\title{
Plasma Alkylresorcinol Metabolite, A Biomarker of Whole-Grain Wheat and Rye Intake, and Risk of Metabolic Syndrome: A Case-Control Study
}

\section{Li Zhou}

Huazhong University of Science and Technology

\section{Xiaoxing Mo}

Huazhong University of Science and Technology

\section{Qiang Wang}

Huazhong University of Science and Technology

Hongjie Liu

Huazhong University of Science and Technology

\section{Yang Liu}

Huazhong University of Science and Technology

\section{Qinchun Luo}

Huazhong University of Science and Technology

Jiawei Yin

Huazhong University of Science and Technology

\section{Xiaobo Peng}

Huazhong University of Science and Technology

\section{Yue Huang}

Huazhong University of Science and Technology

\section{Xiaoqin Li}

Huazhong University of Science and Technology

\section{Zhilei Shan}

Harvard T.H. University of Science and Technology

\section{Guohong Liu}

Shenzhen Center for Disease Control and Prevention

\section{Chao Xia}

Ezhou Center for Disease Control and Prevention

\section{Jinquan Cheng}

Shenzhen Center for Disease Control and Prevention

\section{Wei Yang}

Huazhong University of Science and Technology

\section{Taoping Sun}


Huazhong University of Science and Technology

Liegang Liu ( $\nabla$ lgliu@mails.tjmu.edu.cn )

Huazhong University of Science and Technology Tongji Medical College https://orcid.org/0000-00027029-1224

\section{Original investigation}

Keywords: alkylresorcinol, metabolite, biomarker, whole grains, metabolic syndrome.

Posted Date: June 22nd, 2020

DOl: https://doi.org/10.21203/rs.3.rs-35930/v1

License: (a) This work is licensed under a Creative Commons Attribution 4.0 International License. Read Full License 


\section{Abstract}

Background: Whole-grain intake assessed through dietary recording methods has been suggested to be inversely associated with the metabolic syndrome (MetS) risk in several epidemiological studies. However, limited studies have evaluated the association between whole-grain intake and MetS risk when using objective biomarkers of whole-grain intake. The aim of this study was to examine the association between plasma 3-(3,5-dihydroxyphenyl)-1-propanoic acid (DHPPA), a biomarker of whole-grain wheat and rye intake, and MetS risk in a Chinese population.

Methods: A case-control study of 667 MetS cases and 667 matched controls was conducted based on baseline data of the Tongji-Ezhou Cohort study. Plasma DHPPA concentrations were assessed by highperformance liquid chromatography-tandem mass spectrometry. MetS was determined using definition of the Joint Interim Statement.

Results: Plasma DHPPA was inversely associated with MetS risk. After adjustment for age, sex, body mass index, smoking status, alcohol drinking status, physical activity and education level, the odds ratios (ORs) for MetS across increasing quartiles of plasma DHPPA concentrations were 1 (referent), 0.86 (0.58$1.26), 0.77(0.52-1.15)$, and $0.59(0.39-0.89)$, respectively. The inverse relation between plasma DHPPA and MetS persisted in stratified analyses according to confounding factors. In addition, the cubic spline analysis revealed a potential nonlinear association between plasma DHPPA and MetS, with a steep reduction in the risk at the lower range of plasma DHPPA concentration.

Conclusions: Our study revealed that higher plasma DHPPA concentrations were associated with lower odds of MetS. Our findings provided further evidence to support health benefits of whole grain consumption.

\section{Background}

Recent estimates indicate that the prevalence of metabolic syndrome (MetS) is increasing in China, with an estimated one third of Chinese adults affected (1). MetS is characterized by a cluster of cardiovascular disease risk factors, including central obesity, elevated blood pressure, dyslipidemia, and high blood glucose, and it carries an increased risk of type 2 diabetes and cardiovascular disease $(2,3)$. The pathogenesis of MetS is largely unknown, but probably represents a complex interaction between metabolic, genetic, and environmental factors, which include diet (4).

Whole grain is a good source of dietary fiber, phenolic compounds, phytoestrogens, vitamins, and minerals, which may confer protection on MetS (5). In previous epidemiological studies, whole-grain foods have been associated with lower risk of MetS $(6,7)$. Greater whole-grain intake also was favorably related to markers of MetS such as obesity and dyslipidemia $(8,9)$. However, most epidemiological studies estimated whole-grain intake with the use of dietary recording records (10-12). Given the diversity of whole-grain products in diets, assessing the intake accurately in free-living populations seems to be challenging, and the inaccurate identification of different whole-grain constituents might lead to 
measurement errors $(13,14)$. A more objective estimation of whole-grain intake through use of a biomarker appears to be significant.

Alkylresorcinols are a cluster of phenolic lipids abundant in the outer layer of wheat and rye grain (15). Alkylresorcinols are absorbed through the small intestine, and plasma alkylresorcinols are suggested to be transported in lipoproteins (16). Intact alkylresorcinols and their 2 main end-products- 3,5dihydroxybenozoic acid (DHBA) and 3-(3,5-dihydroxyphenyl)-1-propanoic acid (DHPPA)-are measurable in plasma $(15,17)$. Recently, alkylresorcinols and their metabolites have been suggested as biomarkers of whole-grain rye and wheat intake (18), and they have been used to assess whole-grain rye and wheat intake in several epidemiological studies $(19,20)$. Considering that the half-life of DHPPA (16.3 hours) is significantly longer than that of DHBA (10.1 hours) and alkylresorcinols (5 hours) and blood samples are usually taken after overnight fasting, plasma DHPPA appears to be a good and specific biomarker of whole-grain wheat and rye intake (21-23).

To our knowledge, no study has yet investigated the association of plasma biomarker concentrations of whole-grain wheat and rye intake with MetS risk in a Chinese population. We thus conducted this matched case-control study, which based on the baseline data of the Tongji-Ezhou Cohort (TJEZ) study, to examine the association of plasma DHPPA concentration with MetS.

\section{Methods}

\section{Study population and design}

The TJEZ cohort study is an ongoing prospective cohort study investigating the associations of lifestyle, dietary factors, and genetic markers with chronic diseases. The study was set in Echeng Steel, the largest construction steel production base in Hubei Province, China. All working employees and retired employees from Echeng steel were invited to participate. Participants who were permanent residence in Ezhou and aged above 20 years at the time of recruitment were recruited. Individuals were not recruited if they had cancer or were pregnant. Between 2013 and 2014, 5533 adult residents were recruited, with a response rate of $96.6 \%$. Data allowing the determination of the status of MetS was available for 4137 of the baseline individuals, and the prevalence of MetS was 33.6\%. Among them, we excluded 1966 individuals without plasma DHPPA data, and 97 individuals with severe psychological disorders, physical disabilities, cardiovascular disease, Alzheimer's disease, dementia, tuberculosis, AIDS, or other communicable diseases. Finally, 667 members classified as MetS were included in the analyses. Each MetS case was matched with 1 control individual. Control individuals were randomly selected among participants without MetS in the TJEZ study at baseline, and matching variables including sex and age ( \pm 3 years). The flow chart of participant recruitment and case-control selection is shown in Additional file 1: Figure S1. Ethics approval was granted by the Ethics and Human Subject Committee of Tongji Medical College. All enrolled participants gave written informed consent.

\section{Data collection}


Demographic characteristics and medical history were collected at baseline using standardized, selfadministered questionnaires. Lifestyle factors including smoking habits, alcohol drinking status, and physical activity were also obtained via questionnaires. Individuals who reported smoking $\geq 1$ cigarette/day over the past 6 months were considered as smokers, otherwise, they were defined as nonsmokers. Alcohol drinking status was classified as current drinking ( $\geq 1$ time/week over the previous 6 months) and nondrinking. Physical activity was defined as regular exercise for at least 60 min per week over the previous 6 months. Education level was grouped into three categories: none or elementary school, middle school, and high school or college. Physical examinations including anthropometric measurements were performed by trained nurses. Body weight and standing height were measured in light indoor clothing and without shoes. Waist circumference were measured at the narrowest level over light clothing, using an upstretched tape meter, and measurements were recorded to the nearest $0.1 \mathrm{~cm}$. Body mass index (BMI) was calculated by dividing the weight in kilograms by the square of the height in meters. Resting blood pressure were measured in a seated position after 5 minutes of seated rest. A standard mercury sphygmomanometer was used for obtaining measurements.

After overnight fasting, venous blood samples were collected from all participants at enrollment. All samples were separated for plasma within 1 hour and stored in $-80^{\circ} \mathrm{C}$ freezers until laboratory analysis. Fasting plasma triglycerides, total cholesterol, high-density lipoprotein cholesterol (HDL-C), low-density lipoprotein cholesterol (LDL-C), and glucose were obtained with automated bioassays.

\section{MetS case definition}

MetS was characterized according to the harmonized criteria as the presence of at least three of the following risk factors (24): (1) central obesity: waist circumference $\geq 90 \mathrm{~cm}$ in men or $\geq 80 \mathrm{~cm}$ in women (following Chinese-specific cutoffs for abdominal obesity defined by the International Diabetes

Federation) (25); (2) Hypertriglyceridemia: triglycerides $\geq 1.70 \mathrm{mmol} / \mathrm{L}$; (3) Low levels of HDL-C: HDL-C < $1.03 \mathrm{mmol} / \mathrm{L}$ in men or < $1.30 \mathrm{mmol} / \mathrm{L}$ in women; (4) High blood pressure: blood pressure $\geq 130 / 85$ $\mathrm{mmHg}$ or use of antihypertensive medication; (5) High fasting glucose: $\geq 5.56 \mathrm{mmol} / \mathrm{L}$ or current use of antidiabetic medication or self-reported history of diabetes.

\section{Measurement of plasma DHPPA concentrations}

Plasma DHPPA was measured by high-performance liquid chromatography-tandem mass spectroscopy (LC-MS/MS) (AB Sciex QTRAP 4500, Applied Biosystems, Foster City, CA). Samples from cases and controls were randomly assayed. Prior to analysis, all samples were thawed, mixed thoroughly by vortex. Then, $50 \mu \mathrm{L}$ of plasma sample was spiked with the internal standard ( $1 \mathrm{ng}$ of syringic acid). The sample was hydrolyzed overnight at $37^{\circ} \mathrm{C}$ with $\beta$-glucuronidase/sulfatase and then extracted with acetonitrile. After centrifugation, we collected the supernatant and repeated the procedure once. The combined supernatants were evaporated to dryness under vacuum at $35^{\circ} \mathrm{C}$ and reconstituted with $50 \mu \mathrm{L}$ solvent (acetonitrile/water, 1:1, vol/vol). After centrifugation $20,238^{\prime} g$ at $4^{\circ} \mathrm{C}$ for $5 \mathrm{~min}, 5 \mu \mathrm{L}$ of the supernatant was analyzed with LC-MS/MS. Four replicate quality control samples were analyzed in each batch $(\mathrm{n}=$ 48). Both the intra- and interassay coefficients of variation were $<10 \%$. 


\section{Statistical analysis}

General characteristics were presented as means \pm standard deviations for parametrically distributed variables, medians (interquartile ranges) for nonparametrically distributed variables, and percentage for categorical variables. Differences in descriptive characteristics between the case and control groups were explored using Student $t$ test (normal distribution) or Mann-Whitney $U$ test (non-normal distribution) for continuous variables; for categorical variables, the chi-square test was used. Plasma DHPPA concentrations were considered as continuous variable and categorized into quartiles according to their distribution among the controls (quartile $1,<7.74 \mathrm{nmol} / \mathrm{L}$, quartile $2,7.75-12.30 \mathrm{nmol} / \mathrm{L}$, quartile $3,12.31$ $20.97 \mathrm{nmol} / \mathrm{L}$, quartile $4, \geq 20.98 \mathrm{nmol} / \mathrm{L}$ ). Logistic regression models were used to estimate odds ratios (ORs) and 95\% standard deviations (Cls) of MetS in relation to plasma DHPPA concentrations. The regression models were adjusted for age, sex, BMI, current smoking, current alcohol drinking, physical activity and education level. Linear trend $P$-values were estimated by modeling the median value of each plasma DHPPA category as a continuous variable. To further examined the the potential nonlinear relation between plasma DHPPA and MetS, we used a cubic spline regression model with 4 knots at the 20th, 40th, 60th, and 80th percentiles of plasma DHPPA concentrations.

Given that plasma alkylresorcinols-precursor of DHPPA-are transported in lipoprotein (16), the variations in carrier lipoprotein concentrations might result in their difference in plasma DHPPA concentrations. Hence, a new variable-plasma DHPPA / (total cholesterol + triglycerides) index-was generated to adjust for variations in the concentrations of carrier lipoproteins. Similar to plasma DHPPA concentrations, plasma DHPPA / (total cholesterol + triglycerides) index was considered as a continuous variable and categorized into quartiles according to its distribution among the controls (quartile $1,<1.27 \mathrm{nmol} / \mathrm{mmol}$, quartile $2,1.28-2.14 \mathrm{nmol} / \mathrm{mmol}$, quartile $3,2.15-3.62 \mathrm{nmol} / \mathrm{mmol}$, quartile $4, \geq 3.63 \mathrm{nmol} / \mathrm{mmol}$ ). Logistic regression models were fit to estimate odds ratios (ORs) and $95 \%$ standard deviations (Cls) of MetS in relation to plasma DHPPA / (total cholesterol + triglycerides). The regression models were adjusted for age, sex, BMl, current smoking status, current alcohol drinking status, physical activity and education level. Linear trend P-values were estimated by modeling the median value of each plasma DHPPA / (total cholesterol + triglycerides) category as a continuous variable. To further examined the potential nonlinear relation between plasma DHPPA / (total cholesterol + triglycerides) and MetS, we used a cubic spline regression model with 4 knots at the 20th, 40th, 60th, and 80th percentiles of plasma DHPPA / (total cholesterol + triglycerides) index.

To estimate the consistency of the DHPPA-MetS association according to participant characteristics, we conducted stratified analyses by sex, age ( $<50$ and $\geq 50$ years), BMI ( $<24$ and $\left.\geq 24 \mathrm{~kg} / \mathrm{m}^{2}\right)$, current smoking status (yes or no), current drinking status (yes or no) and physical activity (yes or no). Interactions between plasma DHPPA categories and these confounding factors on ORs of MetS were tested using likelihood ratio tests, comparing models with and without multiplicative interaction terms. Statistical analyses were performed with SPSS 23.0 software (SPSS, Inc.) and Stata/SE 12.0 software (StataCorp LP). $P<0.05$ was considered significant. 


\section{Results}

The general characteristics of the study groups are shown in Table 1. Demographic characteristics, lifestyle factors and education level were comparable between the MetS case and control groups. As expected, individuals with MetS had higher waist circumference and blood pressure, higher levels of fasting plasma glucose, and more adverse lipid profiles (lower HDL-C and higher triglycerides levels) than those without MetS. Plasma DHPPA concentrations were lower in MetS cases than in controls $(10.43 \mathrm{nmol} / \mathrm{L}$ [6.70-17.24] vs. $12.35 \mathrm{nmol} / \mathrm{L}$ [7.74-20.97], $P<0.001)$. Similarly, plasma DHPPA / (total cholesterol + triglyceride) indexes were significantly lower in MetS cases than in controls $(1.54 \mathrm{nmol} / \mathrm{mmol}[0.92-2.48]$ vs. $2.13 \mathrm{nmol} / \mathrm{mmol}[1.28-3.62], P<0.001)$. 
Table 1

Characteristics of MetS cases and controls ${ }^{a}$

\begin{tabular}{|c|c|c|c|}
\hline Parameters & Cases $(n=667)$ & Controls $(n=667)$ & $\begin{array}{l}P \\
\text { value }\end{array}$ \\
\hline Male, n (\%) & $411(61.6)$ & $411(61.6)$ & 1.000 \\
\hline Age (years) & $56.74(9.80)$ & $56.36(10.04)$ & 0.475 \\
\hline Body mass index $\left(\mathrm{kg} / \mathrm{m}^{2}\right)$ & $25.79(2.97)$ & $22.80(2.73)$ & $\begin{array}{l}< \\
0.001\end{array}$ \\
\hline $\mathrm{SBP}(\mathrm{mmHg})$ & $145.23(21.38)$ & $133.72(18.88)$ & $\begin{array}{l}< \\
0.001\end{array}$ \\
\hline $\mathrm{DBP}(\mathrm{mmHg})$ & $83.20(11.93)$ & $77.36(10.48)$ & $\begin{array}{l}< \\
0.001\end{array}$ \\
\hline Waist circumference $(\mathrm{cm})$ & $90.02(7.87)$ & $80.47(7.85)$ & $\begin{array}{l}< \\
0.001\end{array}$ \\
\hline Triglycerides (mmol/L) & $1.87(1.40-2.48)$ & $1.13(0.81-1.47)$ & $\begin{array}{l}< \\
0.001\end{array}$ \\
\hline Total cholesterol (mmol/L) & $4.94(4.29-5.63)$ & $4.69(4.10-5.39)$ & $\begin{array}{l}< \\
0.001\end{array}$ \\
\hline $\mathrm{HDL}-\mathrm{C}(\mathrm{mmol} / \mathrm{L})$ & $1.12(0.93-1.33)$ & $1.42(1.18-1.68)$ & $\begin{array}{l}< \\
0.001\end{array}$ \\
\hline LDL-C (mmol/L) & $2.82(2.30-3.36)$ & $2.63(2.15-3.19)$ & $\begin{array}{l}< \\
0.001\end{array}$ \\
\hline Fasting plasma glucose (mmol/L) & $6.36(1.62)$ & $5.65(0.89)$ & $\begin{array}{l}< \\
0.001\end{array}$ \\
\hline Current smoker, n (\%) & $193(28.9)$ & $220(33.0)$ & 0.110 \\
\hline Current drinker, n (\%) & $195(29.2)$ & $211(31.6)$ & 0.341 \\
\hline Vigorous activity, n (\%) & $265(39.7)$ & $278(41.7)$ & 0.469 \\
\hline Educational level, n (\%) & & & 0.175 \\
\hline None or elementary school & $186(27.9)$ & $162(24.3)$ & \\
\hline Middle school & $294(44.1)$ & $291(43.6)$ & \\
\hline High school or college & $187(28.0)$ & $214(32.1)$ & \\
\hline
\end{tabular}

\footnotetext{
a Data are presented as $\mathrm{n}(\%)$ for categorical data, means (standard deviations) for parametrically distributed data, or medians (interquartile ranges) for nonparametrically distributed data. Abbreviations: DBP, diastolic blood pressure; DHPPA, 3-(3, 5-dihydroxyphenyl)-1-propanoic acid; HDLC, high-density lipoprotein cholesterol; LDL-C, low-density lipoprotein cholesterol; MetS, metabolic syndrome; SBP, systolic blood pressure.
} 


\begin{tabular}{|c|c|c|c|}
\hline Parameters & Cases $(n=667)$ & Controls $(n=667)$ & $\begin{array}{l}P \\
\text { value }\end{array}$ \\
\hline DHPPA (nmol/L) & $\begin{array}{l}10.43(6.70- \\
17.24)\end{array}$ & $\begin{array}{l}12.35(7.74- \\
20.97)\end{array}$ & $\dot{0.001}$ \\
\hline $\begin{array}{l}\text { DHPPA / (total cholesterol + triglycerides) } \\
\mathrm{nmol} / \mathrm{mmol}\end{array}$ & $1.54(0.92-2.48)$ & $2.14(1.28-3.62)$ & $\dot{0} 001$ \\
\hline \multicolumn{4}{|c|}{$\begin{array}{l}\text { a Data are presented as } \mathrm{n}(\%) \text { for categorical data, means (standard deviations) for parametrically } \\
\text { distributed data, or medians (interquartile ranges) for nonparametrically distributed data. } \\
\text { Abbreviations: DBP, diastolic blood pressure; DHPPA, 3-(3, 5-dihydroxyphenyl)-1-propanoic acid; HDL- } \\
\text { C, high-density lipoprotein cholesterol; LDL-C, low-density lipoprotein cholesterol; MetS, metabolic } \\
\text { syndrome; SBP, systolic blood pressure. }\end{array}$} \\
\hline
\end{tabular}

Higher plasma DHPPA concentrations were associated with lower odds of MetS (Table 2). After adjustment for age, sex and $\mathrm{BMI}$, the ORs $(95 \% \mathrm{Cls})$ for quartiles 1 through 4, respectively, of plasma DHPPA concentrations were 1 (referent), $0.86(0.58-1.27), 0.78(0.53-1.15)$, and $0.60(0.40-0.90)$ for MetS. Further adjustment for lifestyle factors and education level did not change the association substantially. After further adjustments for smoking, drinking, physical activity and education level, the ORs ( $95 \% \mathrm{Cls}$ ) of MetS across increasing quartiles of plasma DHPPA concentrations were 1 (referent), $0.86(0.58-1.26), 0.77(0.52-1.15)$, and $0.59(0.39-0.89)$, respectively. Similarly, plasma DHPPA / (cholesterol + triglycerides) indexes also were inversely associated with odds of MetS (see Additional file 2. Table S1). In addition, we further conducted analyses stratified by sex, age, BMI, smoking status, alcohol drinking status and physical activity (Table 3). The inverse relationship between plasma DHPPA and MetS was consistently observed in all subgroups, and tests for multiplicative interaction were not statistically significant in this study $(P>0.05)$. 
Table 2

Associations of plasma DHPPA concentration with MetS ${ }^{\text {a }}$

\begin{tabular}{|c|c|c|c|c|c|}
\hline \multirow[t]{2}{*}{ Variables } & \multicolumn{4}{|c|}{ Quartile of plasma DHPPA concentrations (nmol/L) } & \multirow{2}{*}{$\begin{array}{l}P \text { for } \\
\text { trend }^{b}\end{array}$} \\
\hline & $\begin{array}{l}\text { Q1(referent), < } \\
7.74\end{array}$ & $\begin{array}{l}\text { Q2,7.75- } \\
12.30\end{array}$ & $\begin{array}{l}\text { Q3, 12.31- } \\
20.97\end{array}$ & Q4, $\geq 20.98$ & \\
\hline \multicolumn{6}{|l|}{ MetS } \\
\hline $\begin{array}{l}\text { No. of } \\
\text { cases/controls, } n / n\end{array}$ & $213 / 169$ & $176 / 164$ & $152 / 169$ & $126 / 165$ & \\
\hline Crude OR & 1 & $\begin{array}{l}0.84(0.63- \\
1.13)\end{array}$ & $\begin{array}{l}0.71(0.53- \\
0.96)\end{array}$ & $\begin{array}{l}0.60(0.44- \\
0.82)\end{array}$ & 0.001 \\
\hline Model $1^{\mathrm{c}}$ & 1 & $\begin{array}{l}0.86(0.58- \\
1.27)\end{array}$ & $\begin{array}{l}0.78(0.53- \\
1.15)\end{array}$ & $\begin{array}{l}0.60(0.40- \\
0.90)\end{array}$ & 0.013 \\
\hline Model $2^{d}$ & 1 & $\begin{array}{l}0.86(0.58- \\
1.26)\end{array}$ & $\begin{array}{l}0.77(0.52- \\
1.15)\end{array}$ & $\begin{array}{l}0.59(0.39- \\
0.89)\end{array}$ & 0.012 \\
\hline
\end{tabular}

a Data are odds ratios ( $95 \%$ confidence intervals). Odds ratios (95\% confidence intervals) for MetS were estimated by conditional logistic regression. Abbreviations: DHPPA, 3-(3, 5-dihydroxyphenyl)-1propanoic acid; MetS, metabolic syndrome; OR, odds ratio; Q, quartile.

${ }^{b}$ Tests for linear trend were conducted by using the median value for each quartile and treating it as a continuous variable in the logistic regression.

${ }^{\mathrm{c}}$ Adjusted for sex, age (years) and body mass index $\left(\mathrm{kg} / \mathrm{m}^{2}\right)$.

d Additionally adjusted for smoking (current, former, and never), drinking (current, former, and never), physical activity (yes or no) and educational level (none or elementary school, middle school, and high school or college). 
Table 3

Stratified analyses of MetS risk and plasma DHPPA concentrations by sex, age, body mass index, current smoking status, current alcohol consumption, and physical activity a

\begin{tabular}{|c|c|c|c|c|}
\hline \multirow[t]{2}{*}{ Groups } & \multicolumn{4}{|c|}{ Quartile of plasma DHPPA concentrations (nmol/L) } \\
\hline & $\begin{array}{l}\text { Q1(referent), } \\
<7.74\end{array}$ & $\begin{array}{l}\text { Q2,7.75- } \\
12.30\end{array}$ & $\begin{array}{l}\text { Q3, 12.31- } \\
20.97\end{array}$ & $\mathrm{Q} 4, \geq 20.98$ \\
\hline \multicolumn{5}{|l|}{ Sex } \\
\hline Male (822) & 1 & $\begin{array}{l}0.84(0.54- \\
1.30)\end{array}$ & $\begin{array}{l}0.71(0.45- \\
1.11)\end{array}$ & $\begin{array}{l}0.71(0.45- \\
1.13)\end{array}$ \\
\hline Female (512) & 1 & $\begin{array}{l}0.70(0.41- \\
1.19)\end{array}$ & $\begin{array}{l}0.67(0.39- \\
1.14)\end{array}$ & $\begin{array}{l}0.51(0.29- \\
0.89)\end{array}$ \\
\hline \multicolumn{5}{|l|}{ Age, years } \\
\hline$<50(371)$ & 1 & $\begin{array}{l}0.46(0.24- \\
0.89)\end{array}$ & $\begin{array}{l}0.44(0.22- \\
0.86)\end{array}$ & $\begin{array}{l}0.39(0.19- \\
0.80)\end{array}$ \\
\hline$\geq 50$ (963) & 1 & $\begin{array}{l}0.97(0.65- \\
1.45)\end{array}$ & $\begin{array}{l}0.85(0.57- \\
1.26)\end{array}$ & $\begin{array}{l}0.75(0.50- \\
1.14)\end{array}$ \\
\hline \multicolumn{5}{|c|}{$\begin{array}{l}\text { Body mass index, } \\
\mathrm{kg} / \mathrm{m}^{2}\end{array}$} \\
\hline$<24(650)$ & 1 & $\begin{array}{l}1.02(0.62- \\
1.67)\end{array}$ & $\begin{array}{l}0.78(0.48- \\
1.27)\end{array}$ & $\begin{array}{l}0.67(0.39- \\
1.14)\end{array}$ \\
\hline$\geq 24(684)$ & 1 & $\begin{array}{l}0.59(0.37- \\
0.95)\end{array}$ & $\begin{array}{l}0.58(0.35- \\
0.94)\end{array}$ & $\begin{array}{l}0.58(0.35- \\
0.94)\end{array}$ \\
\hline
\end{tabular}

Current smoking

0.442

Yes (413)

1

$0.75(0.40-$

$0.62(0.33-$

$P$ value for interaction

0.497

0.121

$\begin{array}{lllll} & & 1.38) & 1.19) & 1.01) \\ \text { No (921) } & 1 & 0.79(0.53- & 0.72(0.48- & 0.68(0.45- \\ 1 & 1.07) & 1.03)\end{array}$

Current drinking

0.538

$\begin{array}{lllll}\text { Yes (406) } & 1 & 0.59(0.31- & 0.59(0.31- & 0.61(0.30- \\ & & 1.10) & 1.10) & 1.23) \\ \text { No (928) } & 1 & 0.87(0.59- & 0.74(0.49- & 0.63(0.42- \\ & & 1.31) & 1.11) & 0.96)\end{array}$

Physical activity

0.468

a Data are odds ratios (95\% confidence intervals). The multivariate model was adjusted for sex, age (years), body mass index $\left(\mathrm{kg} / \mathrm{m}^{2}\right)$, smoking (yes or no), drinking (yes or no), physical activity (yes or no) and educational level (none or elementary school, middle school, and high school or college). Abbreviations: DHPPA, 3-(3, 5-dihydroxyphenyl)-1-propanoic acid; MetS, metabolic syndrome; Q, quartile. 


\begin{tabular}{|c|c|c|c|c|c|}
\hline \multirow[t]{2}{*}{ Groups } & \multicolumn{4}{|c|}{ Quartile of plasma DHPPA concentrations (nmol/L) } & \multirow{2}{*}{$\begin{array}{l}P \text { value for } \\
\text { interaction }\end{array}$} \\
\hline & $\begin{array}{l}\text { Q1(referent), } \\
<7.74\end{array}$ & $\begin{array}{l}Q 2,7.75- \\
12.30\end{array}$ & $\begin{array}{l}\text { Q3,12.31- } \\
20.97\end{array}$ & $\mathrm{Q} 4, \geq 20.98$ & \\
\hline Yes (543) & 1 & $\begin{array}{l}0.64(0.38- \\
1.08)\end{array}$ & $\begin{array}{l}0.77(0.45- \\
1.34)\end{array}$ & $\begin{array}{l}0.47(0.28- \\
0.82)\end{array}$ & \\
\hline No (791) & 1 & $\begin{array}{l}0.92(0.59- \\
1.44)\end{array}$ & $\begin{array}{l}0.65(0.42- \\
1.01)\end{array}$ & $\begin{array}{l}0.78(0.49- \\
1.25)\end{array}$ & \\
\hline \multicolumn{6}{|c|}{$\begin{array}{l}\text { a Data are odds ratios ( } 95 \% \text { confidence intervals). The multivariate model was adjusted for sex, age } \\
\text { (years), body mass index }\left(\mathrm{kg} / \mathrm{m}^{2}\right) \text {, smoking (yes or no), drinking (yes or no), physical activity (yes or } \\
\text { no) and educational level (none or elementary school, middle school, and high school or college). } \\
\text { Abbreviations: DHPPA, 3-(3,5-dihydroxyphenyl)-1-propanoic acid; MetS, metabolic syndrome; Q, } \\
\text { quartile. }\end{array}$} \\
\hline
\end{tabular}

In spline regression models, the OR of MetS decreased significantly with increasing plasma DHPPA at less than $18.99 \mathrm{nmol} / \mathrm{L}$, followed by an approximate plateau (Fig. 1). The nonlinear spline terms were statistically significant $(P=0.024)$, suggesting a potential nonlinear relationship between plasma DHPPA concentrations and MetS. The cubic spline analyses also revealed a nonlinear inverse relationship between plasma DHPPA / (cholesterol + triglycerides) index and MetS, with a drastically decrease of MetS risk at less than $2.84 \mathrm{nmol} / \mathrm{mmol}$ but levelled off after that (see Additional file 3. Figure S2).

\section{Discussion}

In this matched case-control study, we observed a nonlinear inverse association between plasma DHPPA concentration and MetS risk. The association did not change materially after adjustment for several confounding factors. Additionally, the association remained generally consistent across different subgroups, suggesting a robust relationship between plasma DHPPA and MetS. As far as we are aware, this is the first study to examine the association between plasma DHPPA, a biomarker of whole-grain wheat and rye intake, and risk of MetS.

Our study revealed that higher plasma DHPPA concentration was associated with lower OR of MetS, which also confirmed previous epidemiological studies used dietary recording methods to estimate whole-grain intake (26-29). In the Framingham Offspring Cohort, whole-grain intake assessed via 126item Food frequency questionnaires (FFQs) was inversely related with the prevalence of MetS (26). Sahyoun et al also found that whole-grain intake, which was estimated by using 3-d food records, was favorably associated with the risk of MetS (29). However, Lutsey et al reported no association between whole-grain intake, which assessed through FFQs, and the risk of MetS among an America population (30). Perhaps because the FFQs used in this study containing only 66 items, which might not effectively differentiate whole-grain from refine-grain foods in the food list. Thus, whole grains might be misclassified and then the measurement errors might occur. Moreover, most dietary recording methods estimated overall whole-grain intake instead of each type of whole-grain intake, and different types of whole grains have different nutrient composition (8), which may be differentially related to human health. 
The main source of whole grains is yeast breads, popcorn, and ready-to-eat breakfast cereals in America (31), brown rice and whole-wheat flour in China (32), and whole-grain bread in Scandinavian countries (33). Findings from a previous study indicated that intake of whole-grain wheat was associated with a reduced risk of colorectal cancer, while other types of whole-grain intake were not (34). Therefore, it is important to distinguish between whole grains when investigating their health-promoting effects. Compared with dietary recording methods which usually assess overall whole-grain intake, our study measuring plasma DHPPA as a biomarker of whole-grain wheat and rye intake seems to be more objective and specific.

Given that plasma alkylresorcinols are transported in plasma lipoproteins, the variations in carrier lipoprotein concentrations among individuals might affect their variations in plasma DHPPA concentrations (16). To adjust for the effect of lipoproteins concentrations on the DHPPA-MetS relationship, plasma DHPPA are expressed relative to plasma total cholesterol and triglycerides. Similar with the DHPPA-MetS association, an inverse association between plasma DHPPA / (total cholesterol and triglycerides) index and MetS was observed in our study. The result further confirmed the inverse association between plasma DHPPA and MetS risk.

Several potential mechanisms might explain the inverse association between plasma DHPPA and MetS. As whole grain intake is often companied with many other health behaviors, whole grain intake may be just a lifestyle marker. However, the beneficial effect of whole grain on MetS was not essentially altered by adjustment for known risk factors. Additionally, the inverse association was consistent among individuals with a greater BMI ( $\left.\geq 24 \mathrm{~kg} / \mathrm{m}^{2}\right)$, and among nondrinkers and individuals with higher physical activity levels. It is more likely that the food compounds and biologically active nutrients in whole grains, such as dietary fiber, minerals, vitamins, and phytochemicals, act directly or indirectly to enhance the beneficial effects on MetS risk. A recent meta-analysis found that dietary fiber intervention lead to a higher abundance of fecal Bifidobacterium and Lactobacillus spp. (35), which has been associated with attenuation of metabolic syndrome (36). Moreover, findings from observational studies and clinical trials indicated that increased consumption of vitamin E, magnesium, and potassium was associated with lower risk of MetS and its components (37-41). The protective effects of whole grains on MetS might be partially explain by these nutrients present in whole grains. Other biologically plausible mechanisms to explain the beneficial effects of whole grain intake on MetS include its effects inflammatory factors, and oxidative stress $(42,43)$.

We observed a nonlinear inverse association between plasma DHPPA and MetS in the present study; in the spline regression model, a reduced curve was observed at the lower range of plasma DHPPA concentration, then followed by an approximate plateau. A possible explanation for this effect is that the increased benefits of whole-grain intake may occur among population with insufficient whole-grain intake, and increasing whole-grain intake might not bring further benefits to population with adequate intake. Further studies are needed to illuminate the potential mechanism and to confirm our findings. Persons with MetS are at increased risk of cardiovascular disease and diabetes, thus our findings were consistent with those from two previous meta-analyses that also revealed nonlinear inverse associations 
between whole-grain intake and stroke and diabetes, with most risk reduced at lower range of whole-grain intake $(11,44)$.

Our study has several strengths. Firstly, we objectively determined concentration of plasma DHPPA, which appears to be a biomarker of whole-grain wheat and rye intake and has been used in several studies to assess whole-grain wheat and rye intake $(19,22)$. Compared with dietary recording methods, the measurement of plasma DHPPA concentration seems to be more objective and independent. Secondly, the matched case-control study design minimized the influence of key confounding factors. Thirdly, the use of a cubic spline regression model enabled us to explore the nonlinear relation between plasma DHPPA and MetS.

Despite these strengths, several limitations should also be acknowledged. The case-control study design did not allow us to establish a causal relationship. Also, because of the case-control design, we cannot exclude the likelihood of recall and selection biases. Moreover, considering that there is limited data on dose-response association between plasma DHPPA and estimated whole-grain wheat and rye intake and the lack of dietary information in our study, it was difficult to compare our results with findings from previous studies assessed whole-grain intake using dietary recording methods. Finally, plasma DHPPA is only a biomarker of whole-grain wheat and rye intake, meaning that intakes of other whole grains such as brown rice and corn, which are also consumed in this population, could not be assessed using this biomarker.

\section{Conclusions}

Our findings implied that higher concentrations of plasma DHPPA, reflecting high whole-grain wheat and rye intake, were associated with lower risk of MetS. Given that the MetS is an identifiable and potentially modifiable risk state for both type 2 diabetes and cardiovascular disease, increasing whole-grain intake may reduce the potential untoward effects of carbohydrate on risk of these diseases. Further prospective cohorts are required to confirmed our findings.

\section{List Of Abbreviations}

$\mathrm{BMI}$, body mass index; $\mathrm{Cl}$, confidence interval; DHBA, 3,5-dihydroxybenozoic acid; DHPPA, 3-(3,5dihydroxyphenyl)-1-propanoic acid; FFQ, food frequency questionnaire; HDL-C, high-density lipoprotein cholesterol; LC-MS/MS, high-performance liquid chromatography-tandem mass spectroscopy; LDL-C, lowdensity lipoprotein cholesterol; MetS, metabolic syndrome; OR, odds ratio; TJEZ, Tongji-Ezhou.

\section{Declarations}

\section{Ethics approval and consent to participate}

Approval of the Ethics and Human Subject Committee of Tongji Medical College was obtained before the start of the work. 


\section{Consent for publication}

Not applicable.

\section{Availability of data and materials}

The datasets used and/or analyzed during the current study are available from the corresponding author on reasonable request

\section{Competing interests}

The authors declare that they have no competing interests.

\section{Funding}

This work was funded by the National Natural Science Foundation of China (81773423), the National Natural Science Foundation of China (81903312), the Major International (Regional) Joint Research Project (NSFC 81820108027), the National Key Research and Development Program of China (2017YFC1600500), the Fundamental Research Funds for the Central Universities (2019kfyXMBZ050), Sanming Project of Medicine in Shenzhen (SZSM201511007). The funding sources had no role in the design and conduct of the study; collection, management, analysis, and interpretation of the data; preparation, review, or approval of the manuscript; or decision to submit the manuscript for publication.

\section{Authors' contributions}

Z.L., L.L., and T.S. designed the study. Z.L. analyzed the data and wrote the manuscript. X.M., Q.W., H.L., Y.L., Q.L., J.Y., X.P., Y.H. and X.L. conducted the experiments and performed the data collection. Z.S., G.L., C.X., J.C., and W.Y. commented on drafts and edited the manuscript. L.L. and T.S. had full access to all the data in the study and take responsibility for the integrity of the data and the accuracy of the data analysis. All authors read and approved the final manuscript.

\section{Acknowledgements}

The chief acknowledgement is to the staff based in the involved hospital and the Echeng Steel corporation for assisting with the fieldwork.

\section{Supplementary information}

Additional file 1: Figure S1. Flowchart of the participant recruitment and case-control selection.

Additional file 2: Table S1. Associations of plasma DHPPA / (total cholesterol + triglycerides) index with MetS.

Additional file 3: Figure S2. Association of plasma DHPPA / (total cholesterol + triglycerides) with metabolic syndrome incidence. 


\section{References}

1. Lu J, Wang L, Li M, Xu Y, Jiang Y, Wang W, Li J, Mi S, Zhang M, Li Y, et al. Metabolic Syndrome Among Adults in China: The 2010 China Noncommunicable Disease Surveillance. J Clin Endocrinol Metab. 2017;102(2):507-15.

2. Lusis AJ, Attie AD, Reue K. Metabolic syndrome: from epidemiology to systems biology. Nat Rev Genet. 2008;9(11):819-30.

3. Wilson PW, D'Agostino RB, Parise H, Sullivan L, Meigs JB. Metabolic syndrome as a precursor of cardiovascular disease and type 2 diabetes mellitus. Circulation. 2005;112(20):3066-72.

4. O'Neill S, O'Driscoll L. Metabolic syndrome: a closer look at the growing epidemic and its associated pathologies. Obes Rev. 2015;16(1):1-12.

5. Fardet A. New hypotheses for the health-protective mechanisms of whole-grain cereals: what is beyond fibre? Nutr Res Rev. 2010;23(1):65-134.

6. Malin SK, Kullman EL, Scelsi AR, Haus JM, Filion J, Pagadala MR, Godin JP, Kochhar S, Ross AB, Kirwan JP. A whole-grain diet reduces peripheral insulin resistance and improves glucose kinetics in obese adults: A randomized-controlled trial. Metabolism. 2018;82:111-7.

7. Zhou AL, Hergert N, Rompato G, Lefevre M. Whole grain oats improve insulin sensitivity and plasma cholesterol profile and modify gut microbiota composition in C57BL/6J mice. J Nutr. 2015;145(2):222-30.

8. Hollaender PL, Ross AB, Kristensen M. Whole-grain and blood lipid changes in apparently healthy adults: a systematic review and meta-analysis of randomized controlled studies. Am J Clin Nutr. 2015;102(3):556-72.

9. Harris Jackson K, West SG, Vanden Heuvel JP, Jonnalagadda SS, Ross AB, Hill AM, Grieger JA, Lemieux SK, Kris-Etherton PM. Effects of whole and refined grains in a weight-loss diet on markers of metabolic syndrome in individuals with increased waist circumference: a randomized controlledfeeding trial. Am J Clin Nutr. 2014;100(2):577-86.

10. Newby PK, Maras J, Bakun P, Muller D, Ferrucci L, Tucker KL. Intake of whole grains, refined grains, and cereal fiber measured with 7-d diet records and associations with risk factors for chronic disease. Am J Clin Nutr. 2007;86(6):1745-53.

11. Aune D, Norat T, Romundstad P, Vatten LJ. Whole grain and refined grain consumption and the risk of type 2 diabetes: a systematic review and dose-response meta-analysis of cohort studies. Eur $\mathrm{J}$ Epidemiol. 2013;28(11):845-58.

12. Juan J, Liu G, Willett WC, Hu FB, Rexrode KM, Sun Q. Whole Grain Consumption and Risk of Ischemic Stroke: Results From 2 Prospective Cohort Studies. Stroke. 2017;48(12):3203-9.

13. Westerterp KR, Goris AH. Validity of the assessment of dietary intake: problems of misreporting. Curr Opin Clin Nutr Metab Care. 2002;5(5):489-93.

14. van Dam RM, Hu FB. Are alkylresorcinols accurate biomarkers for whole grain intake? Am J Clin Nutr. 2008;87(4):797-8. 
15. Ross AB, Kamal-Eldin A, Aman P. Dietary alkylresorcinols: absorption, bioactivities, and possible use as biomarkers of whole-grain wheat- and rye-rich foods. Nutr Rev. 2004;62(3):81-95.

16. Linko-Parvinen AM, Landberg R, Tikkanen MJ, Adlercreutz H, Penalvo JL. Alkylresorcinols from whole-grain wheat and rye are transported in human plasma lipoproteins. J Nutr. 2007;137(5):113742.

17. Koskela A, Samaletdin A, Aubertin-Leheudre M, Adlercreutz H. Quantification of alkylresorcinol metabolites in plasma by high-performance liquid chromatography with coulometric electrode array detection. J Agric Food Chem. 2008;56(17):7678-81.

18. Drake I, Sonestedt E, Gullberg B, Bjartell A, Olsson H, Adlercreutz H, Tikkanen MJ, Wirfalt E, Wallstrom P. Plasma alkylresorcinol metabolites as biomarkers for whole-grain intake and their association with prostate cancer: a Swedish nested case-control study. Cancer Epidemiol Biomarkers Prev. 2014;23(1):73-83.

19. Sun T, Rong Y, Hu X, Zhu Y, Huang H, Chen L, Li P, Li S, Yang W, Cheng J, et al. Plasma Alkylresorcinol Metabolite, a Biomarker of Whole-Grain Wheat and Rye Intake, and Risk of Type 2 Diabetes and Impaired Glucose Regulation in a Chinese Population. Diabetes Care. 2018;41(3):440-5.

20. Sun T, Zhang Y, Huang H, Wang X, Zhou L, Li S, Huang S, Xie C, Wen Y, Zhu Y, et al. Plasma alkylresorcinol metabolite, a biomarker of whole-grain wheat and rye intake, and risk of ischemic stroke: a case-control study. Am J Clin Nutr. 2019;109(2):1-7.

21. Aubertin-Leheudre M, Koskela A, Samaletdin A, Adlercreutz H. Responsiveness of urinary and plasma alkylresorcinol metabolites to rye intake in finnish women. Cancers (Basel). 2010;2(2):513-22.

22. Aubertin-Leheudre M, Koskela A, Samaletdin A, Adlercreutz H. Plasma alkylresorcinol metabolites as potential biomarkers of whole-grain wheat and rye cereal fibre intakes in women. $\mathrm{Br} \mathrm{J}$ Nutr. 2010;103(3):339-43.

23. Soderholm PP, Koskela AH, Lundin JE, Tikkanen MJ, Adlercreutz HC. Plasma pharmacokinetics of alkylresorcinol metabolites: new candidate biomarkers for whole-grain rye and wheat intake. Am J Clin Nutr. 2009;90(5):1167-71.

24. Alberti KG, Eckel RH, Grundy SM, Zimmet PZ, Cleeman JI, Donato KA, Fruchart JC, James WP, Loria $\mathrm{CM}$, Smith SC Jr, et al. Harmonizing the metabolic syndrome: a joint interim statement of the International Diabetes Federation Task Force on Epidemiology and Prevention; National Heart, Lung, and Blood Institute; American Heart Association; World Heart Federation; International Atherosclerosis Society; and International Association for the Study of Obesity. Circulation. 2009;120(16):1640-1645.

25. Grundy SM, Cleeman JI, Daniels SR, Donato KA, Eckel RH, Franklin BA, Gordon DJ, Krauss RM, Savage PJ, Smith SC Jr, et al. Diagnosis and management of the metabolic syndrome: an American Heart Association/National Heart, Lung, and Blood Institute Scientific Statement. Circulation. 2005;112(17):2735-52.

26. McKeown NM, Meigs JB, Liu S, Saltzman E, Wilson PW, Jacques PF. Carbohydrate nutrition, insulin resistance, and the prevalence of the metabolic syndrome in the Framingham Offspring Cohort. 
Diabetes Care. 2004;27(2):538-46.

27. Huang L, Wang H, Wang Z, Zhang J, Zhang B, Ding G. Regional Disparities in the Association between Cereal Consumption and Metabolic Syndrome: Results from the China Health and Nutrition Survey. Nutrients. 2019;11(4).

28. Esmaillzadeh A, Mirmiran P, Azizi F. Whole-grain consumption and the metabolic syndrome: a favorable association in Tehranian adults. Eur J Clin Nutr. 2005;59(3):353-62.

29. Sahyoun NR, Jacques PF, Zhang XL, Juan W, McKeown NM. Whole-grain intake is inversely associated with the metabolic syndrome and mortality in older adults. Am J Clin Nutr. 2006;83(1):124-31.

30. Lutsey PL, Steffen LM, Stevens J. Dietary intake and the development of the metabolic syndrome: the Atherosclerosis Risk in Communities study. Circulation. 2008;117(6):754-61.

31. Burgess-Champoux TL, Larson NI, Neumark-Sztainer DR, Hannan PJ, Story MT. Longitudinal and secular trends in adolescent whole-grain consumption, 1999-2004. Am J Clin Nutr. 2010;91(1):1549.

32. Meharg AA, Lombi E, Williams PN, Scheckel KG, Feldmann J, Raab A, Zhu Y, Islam R. Speciation and localization of arsenic in white and brown rice grains. Environ Sci Technol. 2008;42(4):1051-7.

33. Johnsen NF, Frederiksen K, Christensen J, Skeie G, Lund E, Landberg R, Johansson I, Nilsson LM, Halkjaer J, Olsen A, et al. Whole-grain products and whole-grain types are associated with lower allcause and cause-specific mortality in the Scandinavian HELGA cohort. Br J Nutr. 2015;114(4):60823.

34. Kyro C, Skeie G, Loft S, Landberg R, Christensen J, Lund E, Nilsson LM, Palmqvist R, Tjonneland A, Olsen A. Intake of whole grains from different cereal and food sources and incidence of colorectal cancer in the Scandinavian HELGA cohort. Cancer Causes Control. 2013;24(7):1363-74.

35. So D, Whelan K, Rossi M, Morrison M, Holtmann G, Kelly JT, Shanahan ER, Staudacher HM, Campbell $\mathrm{KL}$. Dietary fiber intervention on gut microbiota composition in healthy adults: a systematic review and meta-analysis. Am J Clin Nutr. 2018;107(6):965-83.

36. Wang J, Tang H, Zhang C, Zhao Y, Derrien M, Rocher E, van-Hylckama Vlieg JE, Strissel K, Zhao L, Obin $\mathrm{M}$, et al. Modulation of gut microbiota during probiotic-mediated attenuation of metabolic syndrome in high fat diet-fed mice. ISME J. 2015;9(1):1-15.

37. Barbagallo M, Dominguez LJ, Galioto A, Ferlisi A, Cani C, Malfa L, Pineo A, Busardo A, Paolisso G. Role of magnesium in insulin action, diabetes and cardio-metabolic syndrome X. Mol Aspects Med. 2003;24(1-3):39-52.

38. Waniek S, di Giuseppe R, Plachta-Danielzik S, Ratjen I, Jacobs G, Koch M, Borggrefe J, Both M, Muller HP, Kassubek J, et al. Association of Vitamin E Levels with Metabolic Syndrome, and MRI-Derived Body Fat Volumes and Liver Fat Content. Nutrients. 2017;9(10).

39. Ley SH, Hanley AJ, Sermer M, Zinman B, O'Connor DL. Lower dietary vitamin E intake during the second trimester is associated with insulin resistance and hyperglycemia later in pregnancy. Eur $\mathrm{J}$ Clin Nutr. 2013;67(11):1154-6. 
40. Sun K, Su T, Li M, Xu B, Xu M, Lu J, Liu J, Bi Y, Ning G. Serum potassium level is associated with metabolic syndrome: a population-based study. Clin Nutr. 2014;33(3):521-7.

41. Tal B, Sack J, Yaron M, Shefer G, Buch A, Ben Haim L, Marcus Y, Shenkerman G, Sofer Y, Shefer L, et al. Increment in Dietary Potassium Predicts Weight Loss in the Treatment of the Metabolic Syndrome. Nutrients. 2019;11(6).

42. Roager HM, Vogt JK, Kristensen M, Hansen LBS, Ibrugger S, Maerkedahl RB, Bahl MI, Lind MV, Nielsen $\mathrm{RL}$, Frokiaer $\mathrm{H}$, et al. Whole grain-rich diet reduces body weight and systemic low-grade inflammation without inducing major changes of the gut microbiome: a randomised cross-over trial. Gut. 2019;68(1):83-93.

43. Murtaza N, Baboota RK, Jagtap S, Singh DP, Khare P, Sarma SM, Podili K, Alagesan S, Chandra TS, Bhutani KK, et al. Finger millet bran supplementation alleviates obesity-induced oxidative stress, inflammation and gut microbial derangements in high-fat diet-fed mice. Br J Nutr. 2014;112(9):1447-58.

44. Aune D, Keum N, Giovannucci E, Fadnes LT, Boffetta P, Greenwood DC, Tonstad S, Vatten LJ, Riboli E, Norat T. Whole grain consumption and risk of cardiovascular disease, cancer, and all cause and cause specific mortality: systematic review and dose-response meta-analysis of prospective studies. BMJ. 2016;353:i2716.

\section{Figures}




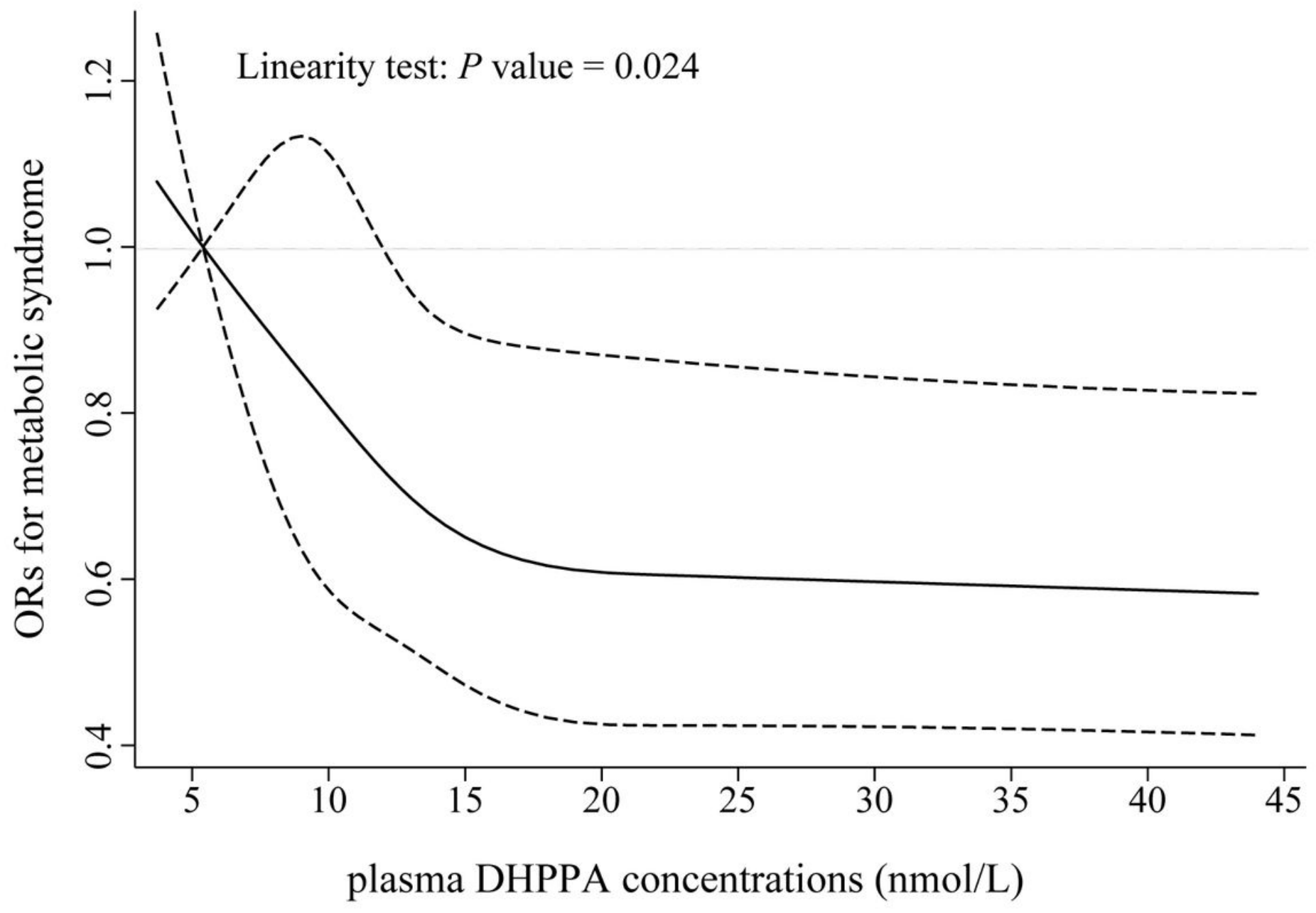

Figure 1

Association of plasma DHPPA concentrations with metabolic syndrome incidence. The restricted cubic spline regression was performed with the use of four knots (20th, 40th, 60th, and 80th percentiles of plasma DHPPA concentrations), and adjusted for sex, age, body mass index, smoking status, alcohol drinking status, physical activity, and education level. Solid lines represent odds ratios and dashed lines represent 95\% confidence intervals. DHPPA, 3-(3, 5-dihydroxyphenyl)-1-propanoic acid; OR, odds ratio.

\section{Supplementary Files}

This is a list of supplementary files associated with this preprint. Click to download.

- Additonalfile1.jpg

- Additionalfile2.docx

- Additioanlfile3.jpg 